\title{
INVARIANCE OF MINIMAL PRIME IDEALS UNDER DERIVATIONS
}

\author{
C.-L. CHUANG AND T.-K. LEE
}

(Communicated by Maurice Auslander)

\begin{abstract}
We construct a counterexample to refute the following conjecture by I. N. Herstein: Is every minimal prime ideal of a semiprime ring invariant under any derivation of this ring?
\end{abstract}

The following conjecture was raised by I. N. Herstein and, recently, has been often mentioned in the literature. See, for example, [4, 8, 9].

Conjecture. If $P$ is a minimal prime ideal of a semiprime ring $R$, is $\delta(P) \subseteq P$ for every derivation $\delta$ of $R$ ?

The primary objective of this note is to refute the conjecture in general. However, the motivation behind this conjecture is to be able to reduce questions regarding derivations in semiprime rings to questions about prime rings and thus the real concern about a given semiprime ring is whether the intersection of the minimal prime ideals invariant under a given derivation is 0 or not. This problem is also due to $\mathrm{I}$. N. Herstein and was listed as the second open question in [9]. In the counterexample constructed here, there is only one minimal prime ideal that is not invariant under a derivation and the intersection of all the minimal prime ideals invariant under this given derivation is still 0 . Thus it remains open as to whether the intersection of all the minimal prime ideals invariant under a given derivation is always 0 or not.

Before proceeding to the construction of our counterexample, it might be interesting to review some positive partial results of the conjecture. There are mainly two directions; one is by restricting the characteristic and the other is by restricting the nilpotency index. For the direction of restricting the characteristic, Krempa [7] proved that any minimal prime ideals of a semiprime algebra over a field of characteristic 0 are always invariant under derivations. Actually, a much better result in this direction is contained in Propositions 1.1 and 1.3 of [5]. Specifically, the following are shown in [5]:

(1) If $P$ is a minimal prime ideal of a ring $R$ such that $R / P$ has characteristic 0 , then $P$ is invariant under any derivations of $R$.

Received by the editors January 5, 1990 and, in revised form, August 23, 1990.

1980 Mathematics Subject Classification (1985 Revision). Primary 16A72, 16A12.

Key words and phrases. Prime ideal, derivation. 
(2) Let $N$ be the prime radical of $R$. If either $R$ or $R / N$ is torsionfree, then all minimal prime ideals of $R$ are invariant under any derivations of $R$. Note that the rings $R$ in (1) and (2) above are not assumed to be semiprime. For the direction of restricting nilpotency index, the second paragraph of the proof of Theorem $\mathrm{B}$ of [8] proves the conjecture for commutative semiprime rings. (Observe that the assumption there on the characteristic is not needed for the invariance of minimal prime ideals under derivations.) It is obvious that a commutative ring is semiprime if and only if it does not have nonzero nilpotent elements. Using essentially the same argument, we can prove the conjecture for any rings without nonzero nilpotent elements. Namely, we can show that any minimal prime ideals of a ring without nonzero nilpotent elements must be invariant under any derivations of the ring. The proof is as follows: For a ring $R$ without nonzero nilpotent elements, the following two facts are well known: (1) A prime ideal $P$ of $R$ is a minimal prime ideal of $R$ if and only if $R \backslash P$ is a maximal multiplicatively closed subset not containing 0 . (2) A nonzero element $a \in R$ is in a given minimal prime ideal $P$ of $R$ if and only if $a m=m a=0$ for some $m \in R \backslash P$. (See, e.g., [1] or [6, Theorem 1.1.1, p. 4].) Now let $P$ be a minimal prime ideal of $R$, and let $a$ be any element of $P$. Then $a m=m a=0$ for some $m \in R \backslash P$. For any given derivation $\delta$ of $R, 0=\delta(a m)=\delta(a) m+a \delta(m)$. Multiplying the identity $\delta(a) m+a \delta(m)=0$ by $\delta(a) m$ on the left-hand side and using the identity $m a=0$, we have $(\delta(a) m)^{2}=0$. Since $R$ does not have nonzero nilpotent elements, $\delta(a) m=0$. Similarly, we can show $m \delta(a)=0$. By the fact (2) cited above, $\delta(a) \in P$. We have thus shown $\delta(P) \subseteq P$ as desired.

A ring $R$ is said to be of bounded index $m$, if $m$ is the least positive integer such that $x^{m}=0$ for all nilpotent elements $x \in R$. A ring without nonzero nilpotent elements is merely a ring of bounded index 1 . Viewing the result above, it seems natural to ask whether the conjecture still holds for rings of bounded index. Unfortunately, this is false as our example shows. Actually, the following theorem due to Beidar and Mikhalëv ([2] or [3, Theorem 8.16]), which generalizes all the above-mentioned results is the best positive result known to the authors:

Theorem (Beidar and Mikhalëv). Let $R$ be a ring of bounded index $m$ such that the additive order of every torsion element of $R$, if any, is strictly larger than $m$. Then all minimal prime ideals of $R$ are invariant under any derivations of $R$.

Now let us construct our desired counterexample:

Counterexample. For any prime $p>0$, there exists a semiprime ring $R$ of characteristic $p$, which satisfies the standard polynomial identity of degree $2 p$, such that a minimal prime ideal $P$ of $R$ is not invariant under a derivation $\delta$ of $R$.

Construction. For simplicity of notation, let us take $p=3$ as an example. The construction for other primes is similar. Arbitrarily choose a field $\Phi$ of 
characteristic 3 and let $M_{3}(\Phi)$ denote the $3 \times 3$ matrix ring over the field $\Phi$. Set $T$ to be the infinite direct product (or power) of $M_{3}(\Phi)$, that is, $T=\prod_{k=1}^{\infty} T_{k}$, where $T_{k}=M_{3}(\Phi)$ for each $k \geq 1$. Obviously, $T$ satisfies the standard polynomial identity of degree 6 since so does each $T_{k}\left(=M_{3}(\Phi)\right)$. Elements of $T$ can be written in the form $x=\left(x_{1}, x_{2}, \ldots\right)$, where $x_{1}, x_{2}, \cdots \in M_{3}(\Phi)$. Let $a=\left(\begin{array}{lll}0 & 1 & 0 \\ 0 & 0 & 1 \\ 0 & 0 & 0\end{array}\right) \in M_{3}(\Phi)$, and let $\Phi[a]$ be the subring generated by $\Phi$ and $a$. Since $a^{3}=0, \Phi[a]$ is equal to $\Phi+\Phi a+\Phi a^{2}$. Our desired ring $R$ is defined by

$$
\begin{aligned}
R=\left\{x=\left(x_{1}, x_{2}, \ldots\right)\right. & \in T: \text { there exist an integer } m \geq 1 \text { and an } \\
& \text { element } \left.y \in \Phi[a] \text { such that } x_{k}=y \text { for all } k \geq m\right\} .
\end{aligned}
$$

Obviously, $R$ is a subring of $T$ and hence must also satisfy the standard polynomial identity of degree 6 . We show that $R$ is semiprime: Suppose that $c R c=0$, where $c=\left(c_{1}, c_{2}, \ldots\right) \in R$. Let $I$ be the infinite direct sum of $M_{3}(\Phi)$, that is,

$$
I=\bigoplus_{k=1}^{\infty} T_{k}=\left\{x=\left(x_{1}, x_{2}, \ldots\right) \in T: x_{k}=0 \text { from some } k \text { on }\right\}
$$

Obviously, $I$ is a two-sided ideal of $R$. So $c I c=0$. That is, $c_{k} T_{k} c_{k}=0$ for each $k$ and hence $c_{k}=0$ for all $k$. So $c=0$ as desired.

For each $m \geq 1$, define

$$
P_{m}=\left\{x=\left(x_{1}, x_{2}, \ldots\right) \in R: x_{m}=0\right\} .
$$

It is easy to see that any prime ideal of $R$ that does not include $I$ as a subset must be one of $P_{m}$ for some $m \geq 1$. Let $P$ be the ideal of $R$ generated by $I$ and the element $u=(a, a, \ldots) \in R$. (That is, each component of $u$ is equal to $a$.) We show that the only prime ideal of $R$ that includes $I$ as a subset is $P$ : Indeed, since $R / I \simeq \Phi[a]=\Phi+\Phi a+\Phi a^{2}, R / P \simeq \Phi[a] /\left(\Phi a+\Phi a^{2}\right) \simeq \Phi$. So $P$ itself is a prime ideal including $I$. Conversely, suppose that $Q$ is a prime ideal of $R$ including $I$ as a subset. Then $Q / I$ is a prime ideal of $R / I$. But $R / I \simeq \Phi[a]=\Phi+\Phi a+\Phi a^{2}$ and the only prime ideal of $\Phi[a]$ is $\Phi a+\Phi a^{2}$. So $Q$ is the ideal generated by $I$ and $u$ as desired. In particular, we have also shown that $P$ is a minimal prime ideal of $R$, since none of $P, P_{m} \quad(m \geq 1)$ can include each other.

Let $b=\left(\begin{array}{ccc}0 & 0 & 0 \\ 1 & 0 & 0 \\ 0 & -1 & 0\end{array}\right) \in M_{3}(\Phi)$. Then $b a-a b=-1$. Let $d$ be the inner derivation of $M_{3}(\Phi)$ defined by $b$. That is, $d(y)=b y-y b$ for $y \in M_{3}(\Phi)$. Since $d(a)=-1$, we have $d(\Phi[a]) \subseteq \Phi[a]$. Our desired derivation $\delta$ on $R$ is defined by $\delta(x)=\left(d\left(x_{1}\right), d\left(x_{2}\right), \ldots\right)$ for each element $x=\left(x_{1}, x_{2}, \ldots\right) \in R$. Since $d(\Phi[a]) \subseteq \Phi[a], \delta$ is a derivation of $R$. But $\delta(u)=(d(a), d(a), \ldots)=$ $(-1,-1, \ldots) \notin P$. So $\delta(P) \nsubseteq P$. Hence the minimal prime ideal $P$ is not invariant under the derivation $\delta$. 
We conclude this note by briefly indicating how the construction should be modified for arbitrary characteristic $p>0$ : Choose $\Phi$ to be a field of characteristic $p>0$. Let $M_{p}(\Phi)$ denote the $p \times p$ matrix ring over $\Phi$ and let $e_{i j}(1 \leq i, j \leq p)$ denote the matrix unit with 1 in the $(i, j)$-entry and with 0 elsewhere. Define $a=\sum_{i=1}^{p-1} e_{i, i+1}$ and $b=\sum_{i=1}^{p-1} i e_{i+1, i}$. Then $b a-a b=-1$, where $1=\sum_{i=1}^{p} e_{i i}$ denotes the identity matrix of $M_{p}(\Phi)$. Now the rest of the construction is analogous to the case $p=3$.

\section{REFERENCES}

1. V. A. Andrunakievič and Ju. M. Rjabuhin, Rings without nilpotent elements and completely prime ideals, Dokl. Akad. Nauk SSSR 180 (1968), 9-11; English transl. in Soviet Math. Dokl. 9 (1968), 565-568.

2. K. I. Beidar and A. V. Mikhalëv, Orthogonal completeness and minimal prime ideals, Trudy Sem. Petrovski 10 (1984), 227-234.

3. __ Orthogonal completeness and algebraic systems, Uspekhi Mat. Nauk 40 (1985), 79115; English transl. in Russian Math. Surveys 40 (1985), 51-95.

4. Jeffrey Bergen, Automorphic-differential identities in rings, Proc. Amer. Math. Soc. 106 (1989), 297-305.

5. K. R. Goodearl and R. B. Warfield, Jr., Primitivity in differential operator rings, Math. Z. 180 (1982), 503-523.

6. I. N. Herstein, Rings with involution, Univ. of Chicago Press, Chicago, 1976.

7. J. Krempa, Radicals and derivations of algebras, Proc. of Eger Conf., North-Holland, 1982.

8. J. Krempa and J. Matczuk, On the composition of derivations, Rend. Circ. Mat. Palermo (2) 33 (1984), 441-445.

9. Gail Letzter, Derivations and nil ideals, Rend. Circ. Mat. Palermo (2) 37 (1988), 174-176.

Department of Mathematics, National Taiwan University, Taipei, Taiwan 10764, REPUBLIC OF CHINA 